\title{
Mudança na dieta da traíra Hoplias malabaricus (Bloch) (Erythrinidae, Characiformes) em lagoas da bacia do rio Doce devido à introdução de peixes piscívoros
}

\author{
Paulo dos Santos Pompeu ${ }^{1}$ \\ Alexandre Lima Godinho ${ }^{1}$
}

\begin{abstract}
Diet changes of the trahira Hoplias malabaricus (Bloch) (Erythrinidae, Characiformes) due to piscivorous introductions in Rio Doce valley lakes. Two piscivorous fishes, peacock bass (Cichla monoculus Spix \& Agassiz, 1831) (Perciformes) and piranha (Pygocentrus nattereri Kner, 1860) (Characiformes), were introduced in some Rio Doce valley lakes (19 $50^{\circ} \mathrm{S}, 42^{\circ} 40^{\prime} \mathrm{W}$ ) for sport fisheries enhancement. As a consequence, small individuals and species were practically vanished in the host lakes. In this study, the effects of peacock bass and piranha introductions on the diet of a native piscivorous fish, the trahira-Hoplias malabaricus (Bloch, 1794) are presented. Trahira's diet from three lakes were was compared with the stomach contentsdiet of trahira's living in another between three lakes with and three withoutstocked with the piscivorous species peacock bass and piranha. In the lakes with introduced fishes species, the consumption of fish was significantly smaller and this food item have been this item partly replaced by aquatic invertebrates. This shift on of trahira's diet to the low abundance of its original prey, is attributed to the small fishes. This diet plasticity adaptative capacity he diet plasticity detected for trahira might be allowing its maintenance in the lakes with peacock bass and piranha. KEY WORDS. Cichla, Pygocentrus, Hoplias malabaricus, diet, species introduction
\end{abstract}

A introdução de peixes fora de sua distribuição geográfica original é prática antiga e usual. Incremento da pesca, aqüicultura, controle de organismos prejudiciais e ornamentação estão entre as razões mais comuns (COURTENAY \& STAUFFER 1984). As introduções têm causado profundas modificações nas comunidades hospedeiras como eliminação de espécies, remoção da vegetação, introdução de parasitas e doenças, alterações tróficas (COURTENAY \& STAUFFER 1984) e/ou alterações genéticas (FERGUSON 1990). Nos trópicos, pouco se sabe sobre os efeitos de introduções de peixes nas comunidades hospedeiras mas mudanças em larga escala como eliminação de espécies e modificações na estrutura das comunidades hospedeiras, já foram registradas (ZARET \& PAINE 1973; BAREL et al. 1985; MILLER 1989; GodinHO \& Formagio 1992; GodinHO et al. 1994).

Para incrementar a pesca esportiva, o tucunaré (Cichla monoculus Spix \& Agassiz, 1831) e a piranha (Pygocentrus nattereri Kner, 1860) foram introduzidos na década de 1960 no sistema de lagoas do médio rio Doce. O tucunaré é piscívoro de origem amazônica e pode atingir até 15 kg (LOWE-MCCONNELL 1975; Goul-

1) Departamento de Zoologia, Instituto de Ciências Biológicas, Universidade Federal de Minas Gerais. Avenida Antônio Carlos 6627, 31270-901 Belo Horizonte, Minas Gerais, Brasil. E-mail: pompeups@uai.com.br 
DING 1980; ZARET 1980). A piranha atinge tamanhos bem menores e tem distribuição original restrita às bacias Amazônica e do Paraná. É também predadora e tem nos peixes um dos seus principais itens alimentares (RESENDE et al. 1996).

Tanto o tucunaré quanto a piranha ocorrem atualmente em várias lagoas do citado sistema. Na lagoa Dom Helvécio, a maior delas com $6,87 \mathrm{~km}^{2}$ (SAIJO \& TUNDISI 1985), estes peixes foram registrados pela primeira vez no início da década de 1980. Cerca de 10 anos depois, quando essas duas espécies compunham $86 \%$ do número de indivíduos capturados na pesca experimental, pouco mais da metade da riqueza original de peixes já havia desaparecido (GodinHo \& FoRMAGIO 1992). Os peixes de pequeno porte foram praticamente extintos, sendo que a comunidade da lagoa passou a ser composta basicamente por espécies e indivíduos de maior porte (GODINHO \& FoRMAGIO 1992). Situação semelhante foi também detectada em outras duas lagoas da região (GODINHO et al. 1994).

Essa acentuada modificação nas comunidades de peixes com a introdução do tucunaré e da piranha presumivelmente alterou a disponibilidade de presas para as espécies nativas. Este trabalho teve como objetivo detectar os efeitos dessa modificação na dieta de um piscívoro nativo, a traíra Hoplias malabaricus (Bloch, 1794).

O sistema de lagoas do médio rio Doce é composto por cerca de duas centenas de lagoas que ocorrem no Parque Estadual do rio Doce (19 ${ }^{\circ} 50^{\prime} \mathrm{S}, 42^{\circ} 35^{\prime}$ a $42^{\circ} 40^{\prime} \mathrm{W}$ ) (SAIJO \& TUNDISI 1985) e em áreas adjacentes. Duas das lagoas estudadas, Dom Helvécio e Carioca, situam-se dentro da área do parque. Nessas, a vegetação circundante é Mata Atlântica do tipo estacional semidecidual. As demais lagoas estudadas (Jacaré, Ferrugem, Timburé e Palmeiras) estão nas imediações do parque em área onde a vegetação original foi substituída por monoculturas de Eucalyptus. As lagoas apresentam características morfométricas bastante distintas, com área variando de 13 a 687 ha, e profundidade máxima de 9 a 32 m (TUNDISI et al. 1997).

O clima da região é tropical quente, com dois períodos marcantes: chuvoso (outubro-março) e seco (maio-agosto) (TUNDISI 1997). A temperatura média é de $30^{\circ} \mathrm{C}$ e a precipitação anual de $1.500 \mathrm{~mm}$ (NAKAMOTO et al. 1997).

Até quando da realização do presente estudo, o tucunaré e a piranha eram encontrados em três das lagoas estudadas (Dom Helvécio, Jacaré e Carioca), estando ausentes nas demais.

\section{MATERIAL E MÉTODOS}

Os peixes foram capturados com redes de emalhar, armadas na tarde de um dia e retiradas na manhã seguinte. As lagoas foram amostradas uma única véz em julho de 1992 ou julho de 1993, com exceção da Dom Helvécio que foi amostrada em março de 1992 e setembro de 1993.

Em cada lagoa foram armados de três a nove conjuntos de rede, cada um composto por redes das seguintes malhas: $3,4,5,6,7,8,10,12$ e $14 \mathrm{~cm}$, medidas entre nós opostos (malha total). Cada rede possuía $20 \mathrm{~m}$ de comprimento por cerca de $1,6 \mathrm{~m}$ de altura. Os peixes capturados foram fixados em formol $10 \%$ e conservados em álcool a $70 \%$. 
Todas as traíras (H. malabaricus) capturadas foram medidas (comprimento padrão) e dissecadas para a análise do conteúdo estomacal. Os itens alimentares encontrados foram pesados (precisão de $0,01 \mathrm{~g}$ ) e identificados até a menor categoria taxonômica possível. Foram então determinados a frequência de ocorrência $(F i)$ e o peso relativo $(\mathrm{Pi})$ de cada item.

Para cada lagoa, o Índice Alimentar (KAWAKAMI \& VAzzoler 1980) foi calculado para cada item ingerido segundo a fórmula:

$$
\mid A_{i}=\left(F_{i} P_{i}\right) / \sum_{i=1}^{n} F_{i} P_{i}
$$

onde: $\left(\mathrm{IA}_{\mathrm{i}}\right)$ Índice Alimentar do item $\mathrm{i}, \mathrm{F}_{\mathrm{i}}=$ frequência de ocorrência do item $\mathrm{i}, \mathrm{P}_{\mathrm{i}}=$ peso relativo do item $\mathrm{i}, \mathrm{n}=$ número de itens alimentares.

A dieta da traíra foi comparada entre os dois grupos de lagoas estudados: com (Lagoas Dom Helvécio, Jacaré e Carioca) e sem espécies introduzidas (lagoas Palmeiras, Timburé e Ferrugem). Diferenças entre os valores do Índice Alimentar para peixes, presa preferencial dos adultos de traíra, foram testadas entre os dois grupos de lagoas através do teste t. Por serem valores percentuais, os dados do Índice Alimentar foram previamente transformados através do arco seno (FLEISS 1981) para a aplicação do teste.

\section{RESULTADOS}

Foram capturadas 205 traíras, sendo 139 nas lagoas com tucunaré e piranha e 66 nas demais, sendo que, do total, 117 apresentaram algum tipo de conteúdo estomacal. Não foram observadas diferenças na percentagem de indivíduos com conteúdo estomacal por classe de tamanho entre os dois grupos de lagoas (Fig. 1). No entanto, nas lagoas com espécies introduzidas, foi analisado maior número relativo de indivíduos acima de $25 \mathrm{~cm}$, enquanto nas demais lagoas foi analisado de maior número relativo de indivíduos menores que $20 \mathrm{~cm}$.

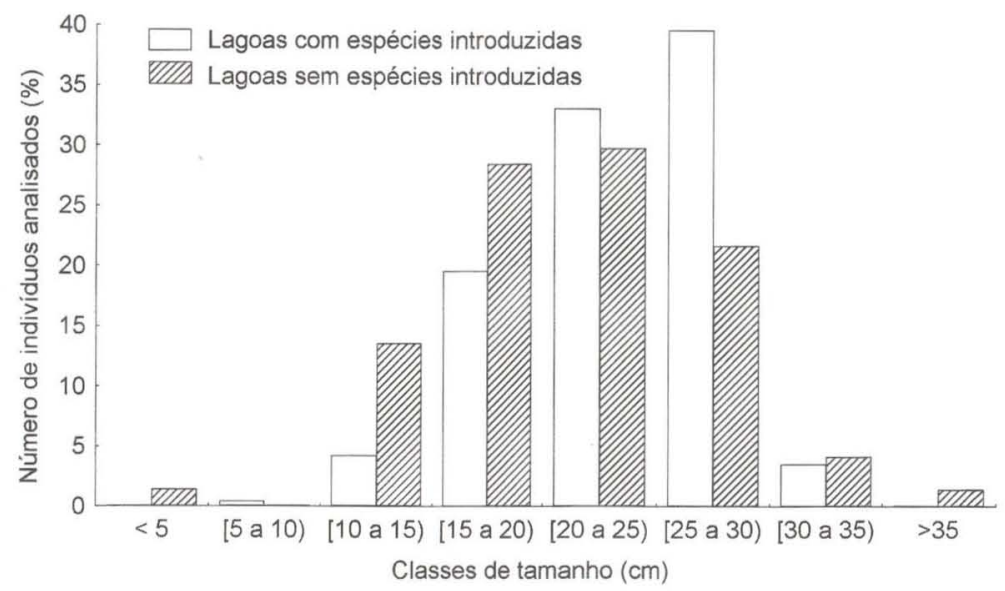

Fig. 1. Número relativo de individuos por classe de tamanho das traíras que apresentaram conteúdo estomacal em lagoas com e sem espécies introduzidas. 
A análise do conteúdo estomacal das traíras revelou que os principais itens ingeridos foram peixes, ninfas de Odonata e camarões (Tab. I). Matéria vegetal e insetos terrestres foram ingeridos em menor proporção. Entre os peixes ingeridos, foram identificadas oito espécies: Lycengraulis sp.; Astyanax bimaculatus (Linnaeus, 1758); Oligosarcus solitarius Menezes, 1987; H. malabaricus (Bloch, 1874); Leporinus steindachneri Eigenmann, 1907; Cyphocharax gilbert (Quoy \& Gaimard, 1824) e Crenicichla lacustris (Castelnau, 1855). Os indivíduos dessas espécies foram, de maneira geral, ingeridos inteiros.

Foram detectadas diferenças nos valores do Índice Alimentar entre os dois grupos de lagoas. Nas lagoas com espécies introduzidas os valores do Índice Alimentar para peixes foram significativamente menores $(\mathrm{t}=-2,80 ; \mathrm{p}=0,049$, $\mathrm{df}$ 4). Nessas lagoas, particularmente na Dom Helvécio e Jacaré, o consumo de peixes foi substituído por invertebrados aquáticos (Fig. 2), principalmente ninfas de Odonata e camarões (Tab. I).

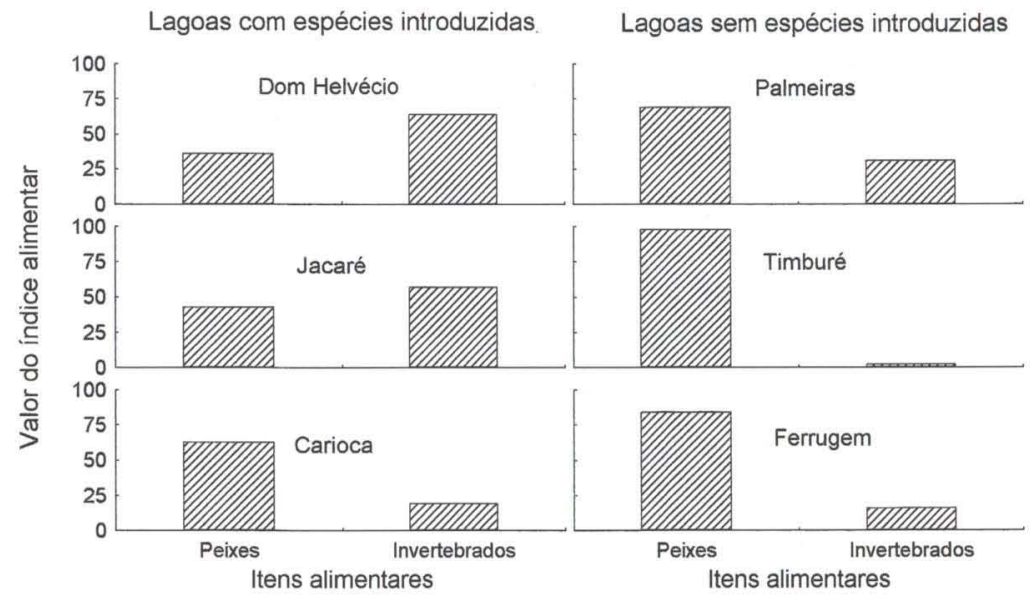

Fig. 2. Índices alimentares (IA) para peixes e invertebrados aquáticos ingeridos pela traíra em lagoas com e sem espécies introduzidas.

Tabela I. Índice Alimentar (IA) dos itens consumidos pela traíra (Hoplias malabaricus) em lagoas do médio rio Doce com e sem piscivoros introduzidos $(n=$ número de estômagos com alimento).

\begin{tabular}{|c|c|c|c|c|c|}
\hline \multirow[t]{2}{*}{ Lagoa } & \multicolumn{5}{|c|}{ Item alimentar } \\
\hline & Peixe & Camarão & Ninfa de Odonata & Inseto terrestre & Matéria vegetal \\
\hline \multicolumn{6}{|l|}{ Com espécies introduzidas } \\
\hline Dom Helvécio $(n=26)$ & 0,36 & 0,24 & 0,40 & $<0,01$ & $<0,01$ \\
\hline Jacaré $(n=44)$ & 0,43 & 0,57 & $<0,01$ & $<0,01$ & $<0,01$ \\
\hline Carioca $(n=14)$ & $<0,01$ & $<0,01$ & 0,18 & $<0,01$ & 0,18 \\
\hline \multicolumn{6}{|l|}{ Sem espécies introduzidas } \\
\hline Palmeiras $(n=2)$ & 0,69 & 0,31 & - & - & - \\
\hline Timburé $(n=10)$ & 0,98 & 0,02 & $<0,01$ & - & - \\
\hline Ferrugem $(n=21)$ & 0,84 & 0,16 & - & - & - \\
\hline
\end{tabular}




\section{DISCUSSÃO}

Os peixes piscívoros têm importante papel na estruturação das comunidades de organismos aquáticos (POWER et al. 1985). Quando um novo predador é introduzido e se torna abundante, ele geralmente afeta a comunidade existente. Os exemplos mais significativos dessas modificações têm sido relatados para a introdução de peixes em lagoas onde a ictiofauna nativa sofre alteração em um curto período de tempo (Hughes 1986). Alguns desses exemplos envolvem o tucunaré. Em alguns ambientes em que foi introduzido, seu estabelecimento provocou prejuízos evidentes para a fauna autóctone, pois o aumento de suas populações se deu em função da redução ou eliminação das espécies nativas (ZARET \& PAine 1973; Godinho et al. 1994; Agostinho \& Júlio 1996). Por outro lado, a introdução da piranha (Pygocentrus nattereri) tem sido menos frequente, com poucos relatos em literatura.

A introdução de piscívoros pode afetar as relações tróficas da comunidade de várias maneiras. As atividades de alimentação do novo predador podem, por exemplo, alterar a abundância e tipo de recurso disponível para as espécies nativas, principalmente aquelas com hábitos alimentares similares (COURTENAY \& STAUFFER 1984). A análise da dieta de $H$. malabaricus, de diferentes bacias, tem revelado que os adultos consomem basicamente peixes (KNÖPPEL 1970; LOWE-MCCONNELL 1975), hábito que se sobrepõe ao das espécies introduzidas nas lagoas do médio rio Doce.

De acordo com WinEMILLER (1989) indivíduos jovens de traíra podem ingerir uma grande quantidade de invertebrados aquáticos. Sob esse aspecto as diferenças na alimentação da traíra observadas entre os dois grupos de lagoas poderiam ser atribuídas à maior frequência de indivíduos jovens analisados nas lagoas sem espécies introduzidas. No entanto, foi exatamente das lagoas onde ocorreu maior ingestão de invertebrados aquáticos que proveio o maior percentual de indivíduos de maior porte.

A acentuada diminuição das espécies e indivíduos de pequeno porte nas lagoas do médio rio Doce, relatada por GodiNHO \& FORMAGIO (1992), é um forte indício da elevada pressão que vem sendo exercida pelos predadores introduzidos. Além disso, das oito espécies de peixes identificadas no conteúdo estomacal das traíras, pelo menos cinco delas parecem ter sido extintas em pelo menos uma das lagoas em que foram introduzidos os novos predadores estudados por GODINHO et al. (1994).

As diferenças observadas na alimentação da traíra entre lagoas com e sem o tucunaré e a piranha devem refletir diferenças na disponibilidade de sua presa original. Apesar de piscívora, a traíra é uma espécie oportunista pois mudanças na sua dieta em função da oferta de alimento já foram detectadas por alguns autores (WINEMILLER 1989; MACHADO-ALLISON 1994). De fato, os peixes neotropicais de água doce parecem ser altamente facultativos na sua alimentação, sendo que muitos deles podem alterar sua guilda trófica, de acordo com a composição específica da comunidade, época do ano e mudanças no ecossistema (LOWE-MCCONNELL 1975; WelCOMME 1985; NiCo \& TAPHORN 1988). No caso 
dos predadores, a disponibilidade de presas é determinante na alimentação (GRIFFITHS 1975). A plasticidade na dieta observada para a traíra, que é uma característica de boa parte dos peixes tropicais (LOWE-MCCONNELL 1975), pode estar contribuindo para garantir a manutenção de suas populações nas lagoas do médio rio Doce com espécies introduzidas.

AGRADECIMENTOS. Ao Instituto Estadual de Florestas e ao programa de pós-graduação em Ecologia, Conservação e Manejo de Vida Silvestre da UFMG pelo apoio à coleta dos peixes, e a FAPEMIG pela bolsa concedida ao primeiro autor.

\section{REFERÊNCIAS BIBLIOGRÁFICAS}

Agostinho, A.A. \& H.F. Julio Jr. 1996. Peixes de outras águas. Ci. Hoje 124: 36-44.

Barel, C.D.N; R. Dorit; P.H. Greenwood. 1985. Destruction of fisheries in Africa's lakes. Nature 315: $19-20$.

Courtenay JR., W.R. \& J.R. Stauffer JR. 1984. Distribution, Biology and Management of Exotic Fishes. Baltimore, Johns Hopkins Univ. Press, 430p.

FERGUSON, M.M. 1990. The genetic impact of introduced fishes on native species. Can. Jour. Zool. 68: 1053-1057.

FLEISS, J.R. 1981. Statistical Methods for Rates and Proportions. New York, John Wiley \& Sons, $2^{\text {nd }}$ ed., $321 \mathrm{p}$.

Godinho, A.L.; M.T. FonseCa \& L.M. ARAúJo. 1994. The ecology of predador fish introductions: the case of Rio Doce valley lakes, p. 77-83. In: R.M. Pinto-Coelho; A. Giani \& E. Sperling (Eds). Ecology and human impact lakes and reservoirs in Minas Gerais with special reference to future development and management strategies. Belo Horizonte, SEGRAC, 193p.

Godinho, A.L. \& P.S. Formagio. 1992. Efeitos da introdução de Cichía ocellaris e Pygocentrus sp. sobre a comunidade de peixes da lagoa Dom Helvécio, MG. In: An. Encontro Anual Aqüicultura Minas Gerais. Belo Horizonte, Vol, 10, p. 93-102.

Goulding, M. 1980. The Fishes and the Forest: Explorations in Amazonian Natural History. Berkeley, Univ. California Press, 280p.

GRIFFITHS, D. 1975. Prey availability and the food of predators. Ecology 56: 1209-1214.

Hughes, N.F. 1986. Changes in the feeding biology of Nile perch, Lates nilotica, (L.) (Pisces: Centropomidae) in Lake Victoria, East Africa, since its introduction in 1960, and its impact on native fish community of Nyasa Gulf. Jour. Fish Biol. 29: 521-548.

KAWAKAMI, E. \& G. VAZOLLER. 1980. Método gráfico e estimativa de índice alimentar aplicado no estudo de alimentação de peixes. Bol. Inst. Oceanogr., S. Paulo, 29: 205-207.

KNoppel, H.A. 1970. Food of central amazonian fishes. Amazoniana 2 (3): 257-352.

Lowe-MCCONNEL, R.H. 1975. Fish communitties in tropical freshwaters. New York, Longman, 337p.

Machado-Allison, A. 1994. Factors affecting fish communities in the flooded plains of Venezuela. Acta Biol. Venez. 15: 59-75.

Miller, D.J. 1989. Introductions and extinction of fish in the African Great lakes. Trends Ecol. Evol. 4 (2): 56-59.

NAKAmoto, N; Y. SAyjo \& J.G. Tundisi. 1997. Metereological environment in the rio Doce valley, p. 51-66. In: J.G. Tundisi \& Y. SAJo (Ed.). Limnological Studies on the rio Doce Valley Lakes, Brazil. São Carlos, Acad. Brasil. Ci., Univ. São Paulo, 528p.

Nico, L.G. \& D.C. TAPHORN. 1988. Food habits of the piranhas in the low llanos of Venezuela. Biotropica 20 (4): 311-321.

Power, M.E.; W.J. MatTEWS \& A.J. STEWART. 1985. Grazing minnows, piscivorous bass, and the stream algae: dynamics of a strong interaction. Ecology 66: 1448-1456. 
Resende, E.K: R.A.C. Pereira; V.L.L. Almeida \& A.G. Silva. 1996. Alimentação de peixes carnívoros da planície alagável do rio Miranda, Pantanal, Mato Grosso do Sul, Brasil. Corumbá, EMBRAPA-CPAP, 36p.

Saijo, Y. \& J.G. Tundisi. 1985. Introduction, p. 3-8. In: Y. SAYjo \& J.G. Tundisi (Eds). Limnological Studies in Central Brazil; Rio Doce Valley Lakes and Pantanal Wetland. Nagoya, Nagoya Univ., 201p.

Tundisi, J.G. 1997. Climate, p. 7-11, In: J.G. Tundisi \& Y. SAIJo (Eds). Limnological Studies on the rio Doce Valley Lakes, Brazil. São Carlos, Acad. Bras. Ci., Univ. São Paulo, 528p.

Tundisi, J.G; T. Matsumura-Tundisi; H. Fukuara; O. Mitamura; S.M. Guillén; R. Henry; O. Rocha; M.C. CALIJURi; M.S.R. IbAÑES; E.L.G. Espíndola \& S. Govoni. 1997. Limnology of fifteen lakes, p. 409-440. In: J.G. Tundisi \& Y. SAIJo. Limnological Studies on the rio Doce Valley Lakes, Brazil. São Carlos, Acad. Bras. Ci., Univ. São Paulo, 528p.

Welcomme, R.L. 1985. River Fisheries. FAO Fish. Tech Pap. 262: 1-330.

WINEMILLER, K.O. 1989. Ontogenetic diet shifts and resource partitioning among piscivorous fishes in the Venezueian Ilanos. Environ. Biol. Fishes 26: 177-199.

ZARET, T.M. \& R.T. PAINE. 1973. Species introduction in a tropical lake. Science 182: 449-455.

1980. Life history and growth relationships of Cichla ocellaris, a predatory South American cichlid. Biotropica 12: 144-157.

Recebido em 27.X11.2000; aceito em 22.X.2001. 